This article was downloaded by: [ ]

On: 02 March 2012, At: 02:22

Publisher: Psychology Press

Informa Ltd Registered in England and Wales Registered Number: 1072954

Registered office: Mortimer House, 37-41 Mortimer Street, London W1T 3J H, UK

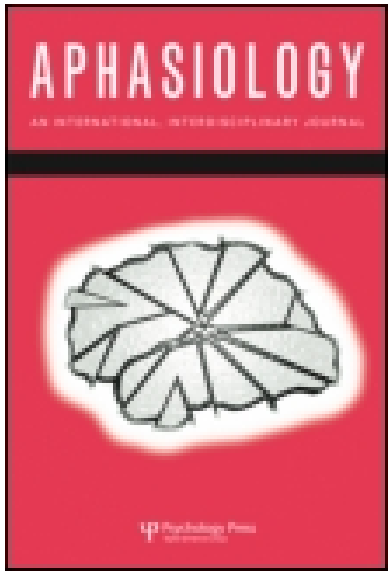

\title{
Aphasiology
}

Publication details, including instructions for authors and subscription information:

http:// www. tandfonline.com/loi/ paph20

\section{How does linguistic knowledge contribute to short-term memory? Contrasting effects of impaired semantic knowledge and executive control}

\author{
Paul Hoffman ${ }^{a}$, Elizabeth J efferies ${ }^{b}$, Sheeba Ehsan ${ }^{a}$, Roy \\ W. J ones ${ }^{c} \&$ Matthew A. Lambon Ralph ${ }^{\text {a }}$ \\ ${ }^{a}$ Neuroscience and Aphasia Research Unit, School of \\ Psychological Sciences, University of Manchester, Manchester, \\ UK \\ ${ }^{\mathrm{b}}$ Department of Psychology, University of York, York, UK \\ ${ }^{c}$ Research Institute for the Care of Older People (RICE), Bath, \\ UK
}

Available online: 01 Aug 2011

To cite this article: Paul Hoffman, Elizabeth J efferies, Sheeba Ehsan, Roy W. J ones \& Matthew A. Lambon Ralph (2012): How does linguistic knowledge contribute to short-term memory? Contrasting effects of impaired semantic knowledge and executive control, Aphasiology, 26:3-4, 383-403

To link to this article: http:// dx.doi.org/ 10.1080/ 02687038.2011.581798

\section{PLEASE SCROLL DOWN FOR ARTICLE}

Full terms and conditions of use: http://www.tandfonline.com/page/terms-andconditions

This article may be used for research, teaching, and private study purposes. Any substantial or systematic reproduction, redistribution, reselling, loan, sub-licensing, systematic supply, or distribution in any form to anyone is expressly forbidden.

The publisher does not give any warranty express or implied or make any representation that the contents will be complete or accurate or up to date. The accuracy of any instructions, formulae, and drug doses should be independently verified with primary sources. The publisher shall not be liable for any loss, actions, 
claims, proceedings, demand, or costs or damages whatsoever or howsoever caused arising directly or indirectly in connection with or arising out of the use of this material. 


\title{
How does linguistic knowledge contribute to short-term memory? Contrasting effects of impaired semantic knowledge and executive control
}

\author{
Paul Hoffman ${ }^{1}$, Elizabeth Jefferies ${ }^{2}$, Sheeba Ehsan ${ }^{1}$, Roy W. Jones ${ }^{3}$, \\ and Matthew A. Lambon Ralph ${ }^{1}$
}

\author{
${ }^{1}$ Neuroscience and Aphasia Research Unit, School of Psychological Sciences, \\ University of Manchester, Manchester, UK \\ ${ }^{2}$ Department of Psychology, University of York, York, UK \\ ${ }^{3}$ Research Institute for the Care of Older People (RICE), Bath, UK
}

\begin{abstract}
Background: Linguistic knowledge makes an important contribution to verbal STM. Some theories, including Baddeley's original conception of the episodic buffer, hold that harnessing linguistic knowledge to support STM is executively demanding. However, some recent evidence suggests that the linguistic contribution does not depend on executive resources.

Aims: In this study we tested the hypothesis that activation of language representations is automatic and that executive control is most important when the material to be remembered is incompatible with this automatic activation.

Methods \& Procedures: Word list recall was tested in three patients with transcortical sensory aphasia (TSA) following stroke. All had preserved word repetition and digit span but poor comprehension associated with impaired executive control. They were compared with two semantic dementia (SD) patients with degraded semantic representations but intact executive control. Patients repeated word lists that varied in their semantic and syntactic resemblance to meaningful sentences.

Outcomes \& Results: The executively impaired TSA patients showed large benefits of semantic and syntactic structure, indicating that their executive deficits did not interfere with the normal linguistic contribution to STM. Instead they showed severe deficits in repetition of scrambled word lists that did not follow usual syntactic rules. On these, the patients changed the word order to better fit their existing knowledge of syntactic structure. In contrast, the SD patients had no problems repeating words in unusual sequences but their semantic knowledge degradation led to frequent phonological errors due to a loss of "semantic binding", the process by which semantic knowledge of words helps to constrain their phonological representation.
\end{abstract}

\footnotetext{
Address correspondence to: Dr Paul Hoffman, School of Psychological Sciences, Zochonis Building, University of Manchester, Oxford Road, Manchester, M13 9PL, UK. E-mail: paul.hoffman@ manchester.ac.uk

We are indebted to the patients and their carers for their generous assistance with this study. PH was supported by a studentship from the University of Manchester and the research was supported by grants from the MRC (G0501632) and NIMH (MH64445).
}

$\begin{array}{rr}\text { C) } 2012 \text { Psychology Press, an imprint of the Taylor \& Francis Group, an Informa business } \\ \text { http://www.psypress.com/aphasiology } & \text { http://dx.doi.org/10.1080/02687038.2011.581798 }\end{array}$


Conclusions: These findings suggest that linguistic support for STM consists of (a) automatic activation of semantic and syntactic knowledge and (b) executive processes that inhibit this activation when it is incompatible with the material to be remembered.

Keywords: Short-term memory; Semantic knowledge; Syntax; Executive control; Sentence repetition

Traditionally, two main approaches to the study of verbal $\mathrm{STM}^{1}$ have been taken. Some researchers have taken the view that STM capacity can be studied as an isolable cognitive system, independently of the language-processing apparatus used in speech comprehension and production. This modular approach has yielded considerable progress, and the most well-known product is probably the Baddeley and Hitch working memory model, which implements verbal STM by means of the phonological loop (Baddeley \& Hitch, 1974). However, in recent years there has been a growing realisation that the language system plays an important part in short-term retention, fuelled by observations such as the effects of psycholinguistic variables like word frequency and imageability on STM span (Bourassa \& Besner, 1994; Hulme et al., 1997; Romani, McAlpine, \& Martin, 2008) and, at the sublexical level, of better memory for nonwords composed of familiar phonological components (Gathercole, Willis, Emslie, \& Baddeley, 1991). Proponents of the modular approach have responded to these findings by positing greater interaction between the STM store and language representations. It has been proposed that linguistic knowledge could influence the phonological store through an episodic buffer (Baddeley, 2000) or by means of a redintegration process that cleans up degraded phonological traces (Hulme et al., 1997; Schweickert, 1993). Other researchers have gone further, claiming that temporary retention of words does not require a specialised store at all and that verbal STM is best viewed as maintenance of activated representations within the language system itself (Acheson \& MacDonald, 2009; Martin \& Saffran, 1997; Patterson, Graham, \& Hodges, 1994; Ruchkin, Grafman, Cameron, \& Berndt, 2003).

The common incidence of STM impairments in aphasic patients provides further evidence for the reliance of STM on the language system. A particularly striking illustration of this is provided by patients with semantic dementia (SD), who suffer from a progressive and eventually profound deterioration in verbal and non-verbal semantic knowledge (Hodges \& Patterson, 2007; Snowden, Goulding, \& Neary, 1989). This comprehension deficit occurs in the context of sparing of the neural apparatus for speech production and phonological processing (Jefferies, Jones, Bateman, \& Lambon Ralph, 2005; Meteyard \& Patterson, 2009; Patterson \& MacDonald, 2006). Indeed, SD patients have normal STM span for digits (which they comprehend well; Jefferies, Patterson, Jones, Bateman, \& Lambon Ralph, 2004) and nonwords (for which comprehension is not an issue; Jefferies et al., 2005). However, their STM span for words is poor, particularly for words with the greatest semantic degradation (Jefferies, Jones, Bateman, \& Lambon Ralph, 2004; Patterson et al., 1994). When repeating lists of words they often make phonological errors in which phonemes from different words become "blended" together (e.g., mint, rug $\rightarrow$ "rint, mug"; Hoffman, Jefferies, Ehsan, Jones, \& Lambon Ralph, 2009; Majerus, Norris, \& Patterson, 2007). These errors indicate that activation of semantic representations plays an important part in STM

\footnotetext{
${ }^{1}$ Throughout this article, we use STM to refer solely to STM for verbal material.
} 
for words and that, without this support from semantic representation, the phonological integrity of the memory trace is compromised (Jefferies, Frankish, \& Lambon Ralph, 2006; Patterson et al., 1994).

Data from SD patients indicate that long-term linguistic knowledge makes a key contribution to short-term verbal retention. However, although this linguistic contribution to verbal STM is now well established, the mechanisms and processes underpinning it are still unclear. One key question is the degree to which the use of linguistic information to perform verbal STM tasks depends on executive control processes. Does the harnessing of linguistic activation to hold information in mind require executive control or does it proceed automatically? In some models attentional control is necessary to maintain activation within the language system (Cowan, 1995; Ruchkin et al., 2003) or to integrate serial order information with language representations (Majerus, 2009). In Baddeley's influential working memory model the contents of the temporary phonological store are integrated with linguistic knowledge in a multi-modal episodic buffer (Baddeley, 2000). This process was initially assumed to require executive control, but this view has been called into question by recent dualtask studies (Baddeley, Hitch, \& Allen, 2009; Jefferies, Lambon Ralph, \& Baddeley, 2004). Baddeley and colleagues investigated the role of executive control in generating the sentence superiority effect: the recall advantage for meaningful sentences over lists of unrelated words, thought to arise from greater linguistic support for sentences (Brener, 1940). Participants recalled sentences and arbitrary word lists while sometimes performing a demanding concurrent task that taxed the central executive. While the concurrent task affected recall for both sentences and lists, the size of the sentence superiority effect remained the same, suggesting that the routine linguistic contribution to STM may not depend on executive control (Baddeley et al., 2009).

In fact, in some circumstances material receiving more support from LTM may be less dependent on executive processes. In another dual-task experiment participants recalled strings of unrelated sentences vs more naturalistic "stories" (Jefferies, Lambon Ralph, et al., 2004). The executive task had a selective effect on the unrelated sentences, with no effect on the stories. Why did unrelated sentences require executive control while stories did not? One possibility is that the arbitrary composition of unrelated sentences conflicted with existing language knowledge and executive control was needed to ensure the material was maintained without interference from linguistic LTM. Because they were broadly consistent with activated semantic knowledge, the coherent stories may have generated less conflict and required fewer executive resources. This view represents a radical departure from the original conception of the episodic buffer. On this view, linguistic knowledge plays an automatic and central role in verbal STM. Executive demands arise not as a consequence of integrating STM and LTM but instead are required in situations where participants are asked to recall material that is incompatible with their existing linguistic and semantic knowledge (e.g., arbitrary sequences of unrelated words or sentences).

In this study we investigated the nature of the linguistic contribution to STM by comparing STM in two sets of patients with contrasting linguistic deficits. We tested memory for word lists in two patients with SD and three individuals with transcortical sensory aphasia (TSA) following stroke. The first thing to note about SD and TSA patients is that their aphasic profiles are superficially rather similar. TSA patients have impaired comprehension but fluent speech output and, critically for the present study, preserved single word repetition (Albert, Goodglass, Helms, Rubens, \& Alexander, 
1981). In addition to preserved single word repetition our TSA patients had preserved digit spans, indicating normal performance for phonologically mediated STM. In this sense they were very similar to SD patients, who produce fluent speech and perform well on number-based STM tasks. As in SD, TSA patients have impaired comprehension and, like SD patients, our TSA cases also had non-verbal semantic deficits, suggesting disruption to central semantic processes (Corbett, Jefferies, Ehsan, \& Lambon Ralph, 2009; Jefferies \& Lambon Ralph, 2006).

Despite these superficial similarities, the underlying cause of the comprehension deficits in these two sets of patients is very different. SD patients suffer from gradual degradation of core semantic representations, leading to a highly stable pattern of semantic deficits in which the same concepts are understood or failed irrespective of the precise task (Garrard \& Carroll, 2006; Jefferies \& Lambon Ralph, 2006; Patterson, Nestor, \& Rogers, 2007; Rogers et al., 2004). Our TSA cases, on the other hand, form part of a larger group of comprehension-impaired aphasic individuals for whom the problem is one of impaired semantic control: a failure in the executive regulation of semantic knowledge, required to ensure that task-relevant information is brought to the fore and irrelevant information inhibited (Badre \& Wagner, 2002; Jefferies \& Lambon Ralph, 2006; Thompson-Schill, D’Esposito, Aguirre, \& Farah, 1997). Unlike SD patients the TSA patients are highly sensitive to the executive demands of semantic tasks. They are often able to comprehend certain concepts when provided with external support, but fail to retrieve the same information in more executively demanding circumstances. For example, they show particularly poor comprehension of ambiguous words, as a result of the executive demands of selecting the correct, contextually appropriate interpretation of the word (Hoffman, Rogers, \& Lambon Ralph, in press; Rodd, Davis, \& Johnsrude, 2005). However, they are often able to successfully retrieve the appropriate meaning when selection demands are reduced by presentation of a sentence that places the word in a specific context, (Hoffman, Jefferies, \& Lambon Ralph, 2010; Noonan, Jefferies, Corbett, \& Lambon Ralph, 2010). Similar results are found in picture naming. Patients often make associative errors, which indicate that some item-specific information has been activated, but they have been unable to settle on the correct response (e.g., squirrel $\rightarrow$ "nuts"; lorry $\rightarrow$ "diesel"). They perform much better when given phonological cues that direct activation away from competing words and toward the correct response (Jefferies, Patterson, \& Lambon Ralph, 2008; Soni et al., 2009). Finally, unlike SD patients the TSA cases show executive deficits in non-semantic tasks like the Wisconsin card-sorting task and solving of abstract mechanical puzzles, suggesting a general impairment of cognitive control (Corbett et al., 2009; Jefferies \& Lambon Ralph, 2006).

To summarise, patients with SD and TSA present with two different forms of semantic deficit. SD patients suffer from damage to core semantic representations, but are able to utilise what remains of their knowledge store effectively. In contrast, in TSA representations are relatively intact, but they have difficulty with the executive processes that ensure that relevant aspects of knowledge are activated for the task or situation in hand.

These differing linguistic impairments, and the relative sparing of speech production and phonological processing in both groups, mean that they provide a useful comparison for probing in more detail the nature of the linguistic contribution to verbal STM. In a recent study we compared verbal STM in SD and TSA patients directly (Jefferies, Hoffman, Jones, \& Lambon Ralph, 2008). Although both groups 
showed poor recall of word lists, they displayed divergent error profiles. In line with damage to semantic representations and the resultant breakdown in "semantic binding" (cf. Patterson et al., 1994), SD patients made frequent phonological errors in which individual phonemes were displaced and transposed, often resulting in nonword responses (e.g., cat, $\log \rightarrow$ "lat, cog"). These errors are consistent with reduced phonological coherence as a result of damage to semantic representations (Jefferies et al., 2006; Patterson et al., 1994). Phonemic errors occurred less frequently in TSA patients. However, these patients made numerous errors in the serial ordering of words in the lists. We argued that these errors, which were virtually absent from the SD patients' responses, were a consequence of the TSA patients' poor executive regulation. As discussed earlier, dual-task studies indicate that executive demands in STM tasks increase when the information to be recalled is incompatible with existing linguistic knowledge (Jefferies, Lambon Ralph, et al., 2004). Arbitrary lists of unrelated words, of the sort used in our study, are very different from most language experience. They have none of the syntactic structure that characterises normal speech, nor do the words share any semantic association or overall message. In these circumstances executive processes are likely to be particularly important for ensuring that the appropriate set of words remain active in memory and are produced in the correct order.

On this view TSA patients have difficulty with the executive demands of repeating arbitrary sequences of unrelated words. A critical prediction is that their STM performance should improve when they are presented with more naturalistic material to repeat, because automatic linguistic activation will be more compatible with the material and thus better able to support recall. In the present study we tested this prediction by asking the patients to repeat lists that varied in their semantic and syntactic resemblance to natural sentences (see Table 1). We assumed that the presentation of any list of words would generate some automatic activation in the language system. For example, processing of a word is likely to generate activation of semantically related words, and processing of a sequence of words is likely to activate potentially compatible syntactic structures. For the naturalistic, sentence-like lists this automatic activation would be largely compatible with the presented material, so we predicted low involvement of executive control in ensuring that the correct sequence of words was available for recall. However, on trials where typical semantic or syntactic constraints were violated, automatic activation of existing knowledge would conflict with the sequence of words to be recalled. In this case executive control would be necessary to inhibit the automatic activation and instead direct attentional resources towards maintaining the novel sequence of words that had been presented. Therefore we expected the TSA patients to perform poorly under these conditions. Although this prediction seems fairly intuitive, it is important to remember that theories which propose that the integration of language and STM is executively demanding (such as the original conception of the episodic buffer) make the opposite prediction: they predict that patients with executive deficits would have difficulty with the naturalistic materials, because they are unable to integrate linguistic knowledge with the contents of the STM store.

We compared our TSA patients to two individuals with SD. There were two main reasons for taking this comparative approach. First, it allowed us to check that the STM profile of the TSA patients was specific to their particular control deficit and was not simply a consequence of having comprehension or STM deficits per se. Second, it allowed us to investigate the effects of damaged semantic representations on recall of word lists vs more naturalistic materials. As in previous studies, we expected SD 
TABLE 1

Examples of experimental stimuli

\begin{tabular}{lll}
\hline & Semantically coherent $(S+)$ & Semantically incoherent $\left(S_{-}\right)$ \\
\hline Grammatically correct $(\mathrm{G}+)$ & $\begin{array}{c}\text { The horse and the cart } \\
\text { carried a heavy load } \\
\text { The heavy the carried horse } \\
\text { load and a cart }\end{array}$ & $\begin{array}{c}\text { The teeth and the pearl followed a } \\
\text { higher sin } \\
\text { The higher the followed teeth sin and } \\
\text { a pearl }\end{array}$ \\
\hline
\end{tabular}

cases to make phonological errors as a result of a reduction in the binding processes by which the meanings of words support their phonological representation (Hoffman et al., 2009; Jefferies et al., 2006; Majerus et al., 2007; Patterson et al., 1994). However, since SD patients have intact executive control we did not expect them to demonstrate any difficulty with lists that violated semantic and syntactic constraints (relative to healthy, age-matched controls). In particular we did not expect to observe any deficits in the serial ordering of words, and in this respect we predicted clear divergence between SD and TSA patients. Our previous study had shown that TSA patients have difficulty recalling lists of words in the correct sequence (Jefferies, Hoffman, et al., 2008). We expected the presence of syntactic structure to reduce the rate of serial order errors in TSA and, conversely, that syntactic violations would exacerbate their difficulties with order memory.

\section{METHOD}

\section{Patients}

Three TSA patients (ME, LS, and PG) were recruited from stroke clubs and clinical referrals in the north-west of England. All had chronic impairments as a result of a CVA at least 1 year previously. They were classified as TSA using criteria from the Boston Diagnostic Aphasia Examination (BDAE; Goodglass, 1983). All had impaired verbal comprehension in the context of spared repetition and relatively fluent speech (although PG's speech was somewhat less fluent than the other two cases; see Table 2). Scanning indicated a large left fronto-temporoparietal lesion in the case of LS, left occipito-temporal lesion for ME, and left frontal and capsular lesion for PG. All three individuals have participated in a number of previous studies as part of a case-series of semantically impaired stroke patients that we have sometimes referred to under the umbrella term "semantic aphasia" (e.g., Corbett et al., 2009; Jefferies \& Lambon Ralph, 2006; Noonan et al., 2010). We have proposed that these cases share a common deficit in control and regulation of semantic knowledge. The full case-series includes semantically impaired patients with a range of speech fluencies and repetition abilities. Since fluent speech production and good repetition were prerequisites for the STM experiment described in the present study, not all patients were suitable to take part. For this reason, in this study we tested only the three patients who conformed to the TSA classification as defined above and were available at the time of testing.

Two SD patients (MT and MB) were recruited from memory clinics in Manchester and Bath. They fulfilled published criteria for SD, in that they had a selective semantic impairment for verbal and non-verbal materials (Hodges, Patterson, Oxbury, \& Funnell, 1992). Scanning in both cases revealed typical atrophy focused on the anterior 
TABLE 2

Background details and neuropsychological assessment

\begin{tabular}{|c|c|c|c|c|c|c|c|}
\hline \multirow[b]{2}{*}{ Test } & \multicolumn{3}{|c|}{$T S A$} & \multicolumn{2}{|c|}{ Sem dem } & \multicolumn{2}{|c|}{ Controls } \\
\hline & $M E$ & $L S$ & $P G$ & $M T$ & $M B$ & Mean & $S D$ \\
\hline Age & 38 & 72 & 61 & 60 & 60 & & \\
\hline Sex & $\mathrm{F}$ & $\mathrm{M}$ & $\mathrm{M}$ & $\mathrm{F}$ & $\mathrm{F}$ & & \\
\hline \multicolumn{8}{|l|}{$B D A E^{\mathrm{a}}$} \\
\hline Comprehension percentile & 33 & 13 & 20 & NT & NT & & \\
\hline Fluency percentile & 100 & 90 & 40 & NT & NT & & \\
\hline Repetition percentile & 100 & 90 & 80 & NT & NT & & \\
\hline \multicolumn{8}{|l|}{ Cambridge Semantic Battery ${ }^{\mathrm{b}}$} \\
\hline Picture naming / 64 & $4^{*}$ & $5^{*}$ & $44^{*}$ & $45^{*}$ & $35^{*}$ & 62.3 & 1.6 \\
\hline $\begin{array}{l}\text { Spoken word-picture matching / } 64 \\
\text { Camel and Cactus Test }\end{array}$ & $50^{*}$ & $37^{*}$ & $58^{*}$ & $57^{*}$ & $48^{*}$ & 63.7 & 0.5 \\
\hline Pictures / 64 & $13^{*}$ & $15^{*}$ & $44^{*}$ & $45^{*}$ & $41^{*}$ & 59.0 & 3.1 \\
\hline Words /64 & $34^{*}$ & $16^{*}$ & $40^{*}$ & $46^{*}$ & $40^{*}$ & 60.7 & 2.1 \\
\hline Category fluency ( 8 categories) & $27^{*}$ & $13^{*}$ & $7^{*}$ & $65^{*}$ & $45^{*}$ & 113.9 & 12.3 \\
\hline \multicolumn{8}{|l|}{ Verbal Short-Term Memory } \\
\hline \multicolumn{8}{|l|}{ Digit $\operatorname{span}^{\mathrm{c}}$} \\
\hline Forwards & 6 & 4 & 6 & 7 & 6 & 6.8 & 0.9 \\
\hline Backwards & 3 & $1^{*}$ & $2^{*}$ & 5 & 6 & 4.7 & 1.2 \\
\hline Sentence Repetition ${ }^{\mathrm{d}} / 10$ & 10 & 9 & 6 & NT & NT & - & - \\
\hline \multicolumn{8}{|l|}{ Executive } \\
\hline Letter fluency $(\mathrm{F}, \mathrm{A}, \mathrm{S})$ & $14^{*}$ & $8^{*}$ & $2^{*}$ & 30 & 20 & 41.1 & 11.6 \\
\hline Wisconsin card-sorting task $/ 6$ & $0^{*}$ & $0^{*}$ & $0^{*}$ & NT & NT & $>1^{\mathrm{f}}$ & \\
\hline Brixton spatial rule attainment task $\mathrm{e}^{\mathrm{e}} / 55$ & $11^{*}$ & $14^{*}$ & $26^{*}$ & 37 & 40 & $>28^{\mathrm{f}}$ & \\
\hline Coloured progressive matrices / 36 & 16 & 16 & 23 & 35 & 32 & $>15^{\mathrm{r}}$ & \\
\hline
\end{tabular}

*Denotes abnormal performance (below published cut-offs or more than two standards deviations below normal mean). TSA = transcortical sensory aphasis; Sem dem $=$ semantic dementia; NT $=$ not tested. ${ }^{a}$ Boston Diagnostic Aphasic Examination (Goodglass, 1983). ${ }^{b}$ From Bozeat et al. (2000). ${ }^{c}$ From Wechsler (1987). ${ }^{\mathrm{d}}$ From the BDAE. ${ }^{\mathrm{e}}$ From P. Burgess and Shallice (1997). ${ }^{\mathrm{f}}$ Minimum scores in healthy population.

temporal lobes, bilaterally. The study received ethical approval from the North-west Multi-centre Ethics Committee.

\section{Background neuropsychological testing}

All five patients completed a battery of tests to assess semantic processing and general cognitive status, which revealed a highly specific semantic deficit in the two SD patients and a pattern of comprehension impairment with more widespread cognitive deficits in the TSA cases (see Table 2). Semantic processing was assessed with the Cambridge 64-item semantic battery (Bozeat, Lambon Ralph, Patterson, Garrard, \& Hodges, 2000), which probes knowledge of the same 64 objects in four different tests: (a) picture naming, (b) matching the spoken word to a picture contained in an array of semantically related distractors, (c) the Camel and Cactus Test (CCT), a semantic association test in which the patient has to match the object to a semantically related item from a choice of four (e.g., does CAMEL go with CACTUS, PINE TREE, SUNFLOWER or ROSE). This test was presented in verbal and pictorial forms. In addition, patients completed verbal fluency tasks for eight semantic categories. These tests revealed impaired verbal and non-verbal semantic processing in all five patients. 
Verbal STM was assessed with forward and backward digit span. Importantly, all cases had forward spans of four or more, indicating preservation of phonological STM capacity. However, backward digit spans were noticeably lower in the TSA cases, reflecting the higher executive demands of repeating in reverse order. The TSA cases also completed a sentence repetition task, which indicated good memory for well-formed sentences.

Tests of executive function indicated clear differences between SD and TSA patients. All three TSA patients were severely impaired when asked to generate words that began with particular letter of the alphabet, a task with high control demands because of the need for an open-ended search of linguistic knowledge. These deficits could not be attributed to speech production difficulties, given the patients' excellent repetition skills. In contrast, the SD patients both fell within the range of age-matched controls (although it is possible that there was some slight reduction in this ability, in line with the progressive degradation of word knowledge in these cases). TSA patients were also impaired on non-verbal executive tests. These indicate that the executive control deficits in these cases are domain-general and not limited to regulation of semantic knowledge. SD patients showed no signs of impairment on these tests.

\section{Materials}

Word lists were created following an orthogonal design that varied the semantic coherence of the words and whether they were presented in a grammatically correct order (see Table 1). For simplicity we refer to all four conditions as "word lists" rather than sentences, and abbreviate semantically coherent lists to $\mathrm{S}+$ and incoherent lists to $\mathrm{S}-$ and the grammatically correct lists to $\mathrm{G}+$ and incorrect lists to $\mathrm{G}-$ - In total there were 120 lists: $30 \mathrm{G}+\mathrm{S}+, 30 \mathrm{G}+\mathrm{S}-, 30 \mathrm{G}-\mathrm{S}+$ and $30 \mathrm{G}-\mathrm{S}-$. The $\mathrm{G}+\mathrm{S}+$ lists were all grammatically correct sentences that were as predictable and meaningful as possible. They contained five content words and between two and four function words. To generate $\mathrm{G}+\mathrm{S}-$ lists we took the syntactic frames from the $\mathrm{G}+\mathrm{S}+$ lists and replaced all of the content words with new words that were unrelated to each other. Each word was replaced with a word of the same phonemic and syllabic length, belonging to the same grammatical class and with a similar lexical frequency. This produced $\mathrm{G}+\mathrm{S}-$ lists that were equivalent in phonological complexity and syntactic structure to the $\mathrm{G}+\mathrm{S}+$ lists, and which contained equally familiar words, but which carried minimal semantic content. To generate $\mathrm{G}-\mathrm{S}+$ and $\mathrm{G}-\mathrm{S}-$ lists we took the $\mathrm{G}+$ lists and randomly scrambled the order of the words.

Stimuli were digitally recorded by a male speaker and assembled electronically. To avoid prosodic cues that might have benefited meaningful or grammatically correct lists, each word was recorded in isolation without intonation. Lists were assembled from these individual word recordings. Words were presented at a standard rate of 1 second for each content word and 0.5 second for each function word.

\section{Design and procedure}

Participants were tested over two sessions. In the first session they received all $30 \mathrm{~S}+$ and $30 \mathrm{~S}-$ lists, with half of the lists of each type presented in $\mathrm{G}+$ form and the remainder in $\mathrm{G}-$. The second session featured the same lists but in the alternative word order condition. Participants were instructed to listen to each sequence of words and as soon as it ended to recall as many of the words as possible, in the exact order in which 
they were presented. Six months after completing the initial experiment, TSA patients were retested with shorter lists containing only three content words. These lists were formed by truncating the original lists. Controls and SD patients were not tested on these easier lists as they were expected to perform at ceiling.

\section{Control participants}

Six healthy individuals with a mean age of 63 and educational level of 12 years were recruited as controls. Age and education did not differ significantly from the patients, $t(9)<1$.

\section{Data analysis}

We were interested in both item memory (how many words were recalled irrespective of serial position) and order memory (how many of those words recalled were in correct serial order). Because lists contained varying numbers of function words and some function words were repeated within lists, the analysis was restricted to content words. Item errors were defined as failures to recall a presented word at all in the response. Changes in inflection (e.g., horse $\rightarrow$ "horses" or travel $\rightarrow$ "travelled") were not classed as errors, as these retained the core meaning of the word. There were five content words in each list, meaning that a participant could receive an error score of $0,0.2,0.4,0.6$, 0.8 , or 1 for each list, depending on the number of words they failed to recall correctly.

Measures of order memory have often been confounded with levels of item recall, since participants who recall more items have more opportunities to recall items out of sequence (see Saint-Aubin \& Poirier, 1999). Since it was likely that our patients would recall fewer words than controls, it was important to ensure that our measure of order memory was independent of item recall. We therefore considered the sequence in which content words were produced, ignoring function words and errors. For each pair of adjacent words we considered whether they were produced in the same sequence in which they appeared in the presented list. Our measure of order errors was the number of incorrectly ordered pairs, expressed as a proportion of the total number of word pairs in the response. So, for example, if a participant recalled four words in total, there were three word pairs and they could receive an error score of $0,0.33,0.67$, or 1 for the list, depending on the number of sequencing errors. This controlled for the fact that participants producing fewer words had less opportunity to produce words out of sequence.

Item and order error data were analysed in each patient individually. Crawford and Howell's (1998) modified $t$-test was used to determine in which conditions each patient was impaired, relative to controls. To test for effects of the semantic and syntactic manipulations, chi-square tests were performed on the number of correct responses vs errors made by each patient in each of the experimental conditions.

We also wanted to directly compare each group of patients with each other and with controls, but the small number of patients precluded a conventional by-participants analysis. Instead we performed ANOVAs in which each list was treated as separate case (following Jefferies, Bott, Ehsan, \& Lambon Ralph, 2011; Jefferies, Hoffman, et al., 2008). For each of the 120 lists in the experiment we calculated the mean error score in the TSA cases, the SD patients, and for controls. Therefore semantic and syntactic coherence were between-lists factors and participant group was a within-lists factor. Item and order errors were considered in two separate ANOVAs. 
Intrusion errors (i.e., any words in the response that did not form part of the presented list) were analysed separately and placed into the following five categories:

1. Real words phonologically related to a target word (e.g., cat $\rightarrow$ "cap").

2. Nonwords phonologically related to a target word (e.g., cat $\rightarrow$ "cag").

3. Semantic errors. This category included clear substitution errors (e.g., The tiny mouse $\rightarrow$ "The little mouse") as well as words that were related to the general topic of the list (e.g., The keeper dived but the player scored a goal $\rightarrow$ "The baker scored the goal after the player fired the shot").

4. Perseverations of words produced in an earlier response.

5. Other responses.

\section{RESULTS}

\section{Item errors}

Item errors for the patients and for the control group are shown in Figure 1. Controls showed almost flawless recall of $\mathrm{G}+\mathrm{S}+$ lists (i.e., meaningful, grammatically correct sentences), but recalled fewer words when the lists had no semantic theme and when they were grammatically incorrect. All of the patients showed this same basic pattern, but with varying levels of impairment. The two SD patients showed a selective impairment for the $\mathrm{G}+\mathrm{S}+$ lists, the lists for which healthy participants benefited most from linguistic knowledge. This is consistent with a reduction in the automatic contribution of semantic knowledge to STM, as a consequence of the degradation of conceptual knowledge in these patients. The TSA patients were more severely impaired across multiple conditions. Chi-square tests performed on each patient's data indicated that all patients recalled more words correctly when the lists were semantically coherent, $\mathrm{S}+$ vs $\mathrm{S}-; \chi^{2}(d f=1)>3.5, p<.06$, and that all except LS performed better for grammatically correct lists, $\mathrm{G}+$ vs $\mathrm{G}-; \chi^{2}(d f=1)>5.3, p<.05$.

To compare the three groups we performed an ANOVA in which each word list was treated as a separate case. This revealed main effects of participant group, $F(2$,

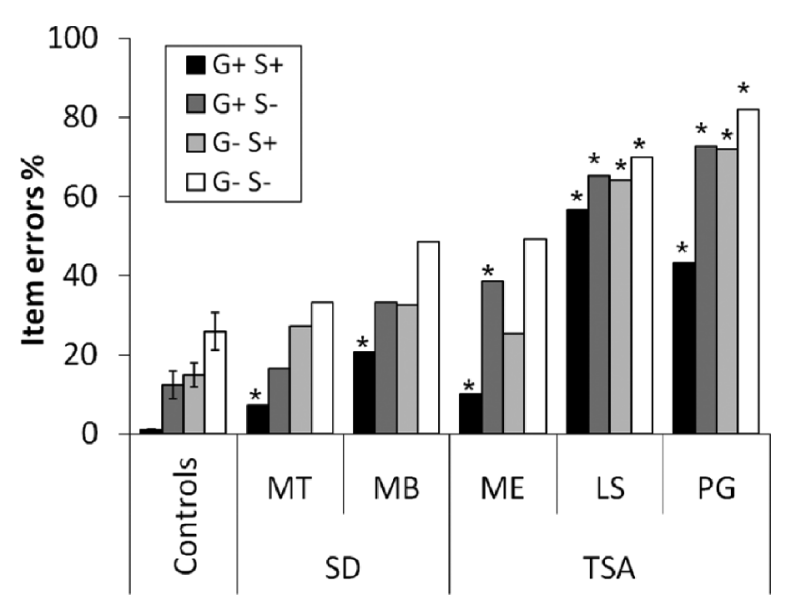

Figure 1. Item errors. *Denotes abnormal error rate; $p<.05$ using Crawford and Howell's modified $t$-test (Crawford \& Howell, 1998). 
$232)=406, p<.001$, semantic coherence, $F(1,116)=74.7, p<.001$, and syntactic structure, $F(1,116)=84.0, p<.001$. Overall there were fewer errors when the lists were semantically coherent (i.e., $\mathrm{S}+$ ) and when the words appeared in a grammatically correct sequence $(\mathrm{G}+)$, reflecting the classic "sentence superiority" effect typically found in STM research (e.g., Brener, 1940; Miller \& Selfridge, 1950). There was also an interaction between semantic coherence and group, $F(2,232)=3.52, p<.05$. Posthoc tests indicated that the presence of semantic coherence improved the performance of the TSA patients more than it did in either the control group or the SD cases: TSA vs controls: $F(1,116)=7.48, p<.01$; TSA vs SD: $F(1,116)=3.99, p<.05$. This suggests that making use of the meaning of a list to support STM is not an executively demanding process. On the contrary, when considered as a group the executively impaired TSA cases were more reliant on the semantic relationships between words, presumably because maintaining a series of unrelated words in memory placed high demands on executive control.

\section{Order errors}

Figure 2 shows the rate of order errors in each patient and in the control group. Within the patient data there was a clear dissociation between SD and TSA patients. SD patients displayed remarkably good order memory, and on the most demanding G-Slists made significantly fewer errors than controls. As predicted, TSA patients made frequent order errors, particularly for $\mathrm{G}-$ lists. All three made significantly more order errors for $\mathrm{G}-$ lists than for $\mathrm{G}+$ lists, $\chi^{2}(d f=1)>4.5, p<.05$. This indicates that TSA patients benefited from the syntactic structure present in the $\mathrm{G}+$ lists, in line with the theory that these materials are less executively demanding because they automatically engage existing representations of familiar syntactic forms that are able to support recall.

The by-lists ANOVA supported this interpretation. There were main effects of participant group, $F(2,232)=148, p<.001$, and syntactic structure, $F(1,116)=75.3$, $p<.001$, but no effect of semantic coherence $(F<1)$. This indicates that participants made use of syntactic structure to recall words in the appropriate order but that the

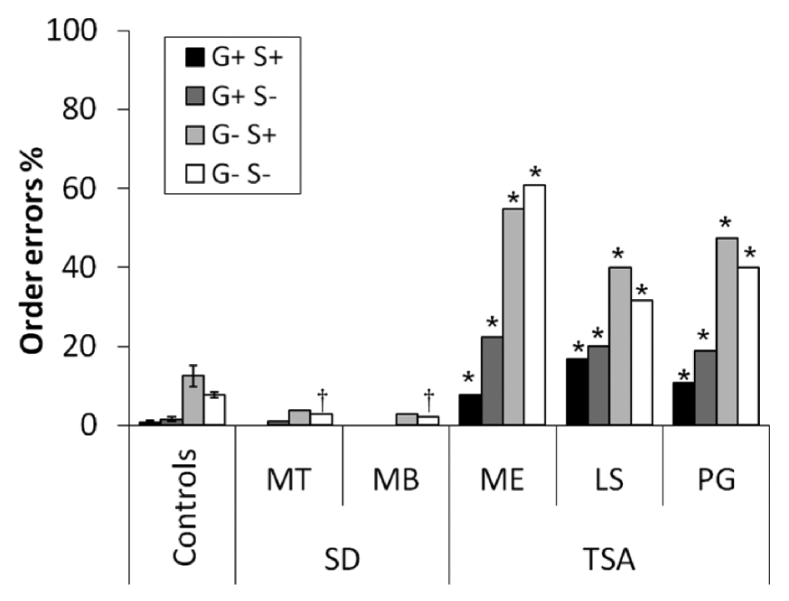

Figure 2. Order errors. *Denotes abnormal error rates; $p<.05$ using Crawford and Howell's modified $t$-test (Crawford \& Howell, 1998). †Denotes significantly fewer errors than controls. 
meaningfulness of the material was not helpful. There was also an interaction between group and syntactic structure, $F(2,232)=34.3, p<.001$, driven by the large syntactic effects in all three TSA patients. Post-hoc tests indicated that the TSA group showed larger syntactic effects than the other two groups: TSA vs controls: $F(1,116)=27.4, p$ $<.01$; TSA vs SD: $F(1,116)=41.2, p<.05$. This is not consistent with the idea that integration of STM with linguistic knowledge is executively demanding. Instead, executive deficits were associated with particular problems in order memory when existing syntactic knowledge was incompatible with the presented material.

\section{Grammatical structure of responses}

TSA patients exhibited particularly poor order memory for lists that violated usual syntactic constraints, which we hypothesised may be due to their inability to inhibit automatic activation as a result of impaired executive control. Inspection of their responses supported this hypothesis. TSA patients often gave grammatically wellformed responses even when the presented list had no syntactic structure at all (see Table 3 for examples). Figure 3 shows the percentage of each patient's responses that were grammatically well formed. SD patients, like healthy controls, gave grammatical responses to $\mathrm{G}+$ sentences, but were able to produce appropriate non-grammatical responses when presented with $\mathrm{G}$ - lists. Conversely, TSA patients showed little sensitivity to the syntactic structure of the list, frequently producing grammatical responses to both types of list - the proportion of grammatical responses to $\mathrm{G}+$ and $\mathrm{G}$ - lists did not differ for any of the TSA cases; $\chi^{2}(d f=1)<2.2, p>.1$. This tendency was most pronounced for patient ME, who could often recall many of the words from a scrambled list correctly but would reconstitute them as a grammatical sentence (e.g., The heavy the carried horse load and a cart $\rightarrow$ "The horse and cart carried a heavy load"). These responses were made without hesitation and without any awareness on the part of the patient that they were anything but verbatim reproductions.

\section{Intrusion errors}

The breakdown of erroneous responses made by each patient (and controls) is shown in Figure 4. The majority of errors made by controls were phonologically related to one of the presented words. Similarly, SD patients made mostly phonological errors, but they were much more likely to produce nonword responses, consistent with poor knowledge of the lexical status of words as a consequence of degraded semantic representations (see Table 3 for examples). The three TSA patients showed different tendencies. Phonological errors were a less-prominent feature of their responses and, like healthy controls, they made hardly any nonword errors. This suggests that the automatic contribution of semantic knowledge to phonological integrity was relatively intact in this group, in line with the theory that their semantic deficits are a result of impaired control processes rather than degraded representations. Instead, perseverative errors were common in all three patients, reflecting a failure of executive control processes needed to inhibit earlier responses (Hamilton \& Martin, 2005). In addition, PG and ME made a substantial number of semantic errors. These errors are consistent with the idea that recall in these patients is strongly influenced by activation of preexisting semantic associations, even when these are inconsistent with the presented material. 
TABLE 3

Examples of patient responses

\begin{tabular}{|c|c|c|c|c|}
\hline Group & Patient & Condition & Presented list & Response \\
\hline \multirow[t]{3}{*}{ TSA } & \multirow[t]{3}{*}{ LS } & $\mathrm{G}+\mathrm{S}+$ & $\begin{array}{l}\text { The keeper dived but the } \\
\text { player scored a goal }\end{array}$ & $\begin{array}{l}\text { The baker scored the goal after } \\
\text { the player fired the shot }\end{array}$ \\
\hline & & $\mathrm{G}+\mathrm{S}-$ & $\begin{array}{l}\text { The college and the husband } \\
\text { advanced a beautiful } \\
\text { subject }\end{array}$ & $\begin{array}{l}\text { The beautiful subject advanced } \\
\text { the college about it }\end{array}$ \\
\hline & & $\mathrm{G}-\mathrm{S}+$ & $\begin{array}{l}\text { Clearly bath a beggar the hot } \\
\text { needed }\end{array}$ & $\begin{array}{l}\text { Hot needed the beggar and } \\
\text { turned it around }\end{array}$ \\
\hline \multirow[t]{3}{*}{ TSA } & \multirow[t]{3}{*}{ ME } & $\mathrm{G}+\mathrm{S}+$ & $\begin{array}{l}\text { The criminal escaped but the } \\
\text { police found a clue }\end{array}$ & $\begin{array}{l}\text { The police found a clue as the } \\
\text { criminal escaped }\end{array}$ \\
\hline & & $\mathrm{G}-\mathrm{S}+$ & $\begin{array}{l}\text { Tight the ship captain and a } \\
\text { crew the ran }\end{array}$ & $\begin{array}{l}\text { The crew and the captain ran a } \\
\text { tight ship }\end{array}$ \\
\hline & & G-S- & $\begin{array}{l}\text { Learned the and homeland } \\
\text { sapphire the sailors good }\end{array}$ & $\begin{array}{l}\text { The soldier and the sapphires } \\
\text { learnt and sailed }\end{array}$ \\
\hline \multirow[t]{3}{*}{ TSA } & \multirow[t]{3}{*}{$\mathrm{PG}$} & $\mathrm{G}+\mathrm{S}+$ & $\begin{array}{l}\text { The artist sketched the } \\
\text { picture while the model } \\
\text { posed }\end{array}$ & $\begin{array}{l}\text { The model posed the artist } \\
\text { sketched }\end{array}$ \\
\hline & & G-S+ & $\begin{array}{l}\text { Slowly cheese nibbled mouse } \\
\text { the tiny the }\end{array}$ & The tiny mouse stole the cheese \\
\hline & & G-S- & $\begin{array}{l}\text { Music the yelled while } \\
\text { airport the staffed the } \\
\text { mirror }\end{array}$ & Staff music lure \\
\hline \multirow[t]{3}{*}{ SD } & \multirow[t]{3}{*}{ MT } & $\mathrm{G}+\mathrm{S}_{-}$ & $\begin{array}{l}\text { The hat drew the ignorant } \\
\text { worm and scars }\end{array}$ & $\begin{array}{l}\text { The pat drew ignorant words } \\
\text { and scars }\end{array}$ \\
\hline & & G-S+ & $\begin{array}{l}\text { Balls the threw quickly the } \\
\text { juggler clever }\end{array}$ & $\begin{array}{l}\text { The balls threw quickly the } \\
\text { juggler never }\end{array}$ \\
\hline & & $\mathrm{G}-\mathrm{S}-$ & $\begin{array}{l}\text { Hook the mortar but dawned } \\
\text { a viewed taxi the }\end{array}$ & $\begin{array}{l}\text { But the daughter a feud the taxi } \\
\text { the }\end{array}$ \\
\hline \multirow[t]{3}{*}{ SD } & \multirow[t]{3}{*}{ MB } & $\mathrm{G}+\mathrm{S}+$ & $\begin{array}{l}\text { The disputed penalty } \\
\text { probably decided the } \\
\text { match }\end{array}$ & $\begin{array}{l}\text { The dispudden penated slightly } \\
\text { discovered the match }\end{array}$ \\
\hline & & $\mathrm{G}+\mathrm{S}_{-}$ & $\begin{array}{l}\text { The citizens slid but the } \\
\text { nature asked a curse }\end{array}$ & $\begin{array}{l}\text { The citizens slipped but the nay } \\
\text { asked a curr }\end{array}$ \\
\hline & & G-S- & $\begin{array}{l}\text { And banner a glove favoured } \\
\text { fine the cloth the }\end{array}$ & $\begin{array}{l}\text { And banger a glove and final a } \\
\text { cloth }\end{array}$ \\
\hline
\end{tabular}

$\overline{\mathrm{G}+\text { and } \mathrm{G}-\text { refer to grammatically correct and incorrect lists; S+ and S- refer to semantically coherent and }}$ incoherent lists.

\section{Replication with shorter lists}

Thus far, clear differences have emerged between TSA and SD patients, particularly with respect to memory for word order. However, it was also the case that the two SD patients displayed higher levels of item recall than two of the TSA cases (PG and LS). This raises the question of whether these individuals would continue to display the same unusual effects on an easier task for which item recall levels were similar to the SD patients. To address this potential issue we retested the TSA patients with shorter lists that contained only three content words. Figure 5 shows that the item error rates were smaller for each patient with these new lists. ME, who performed at a similar level to the SD cases on the original lists, correctly recalled over $80 \%$ of the words in every condition. LS and PG made around $40 \%$ errors on the hardest G-S-condition, which is comparable to the performance of the SD cases on the original lists. Figure 6 


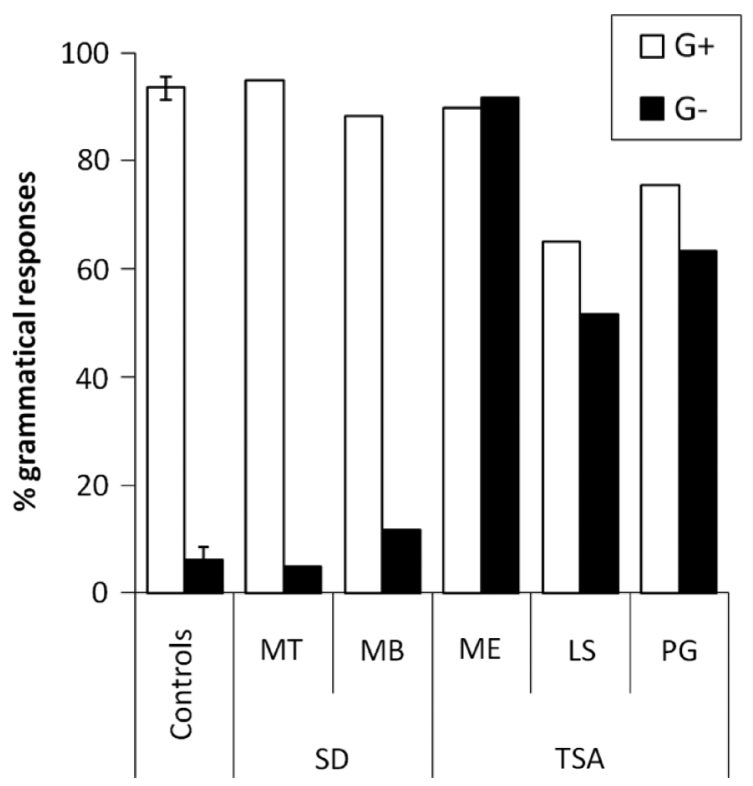

Figure 3. Grammatical structure of responses.

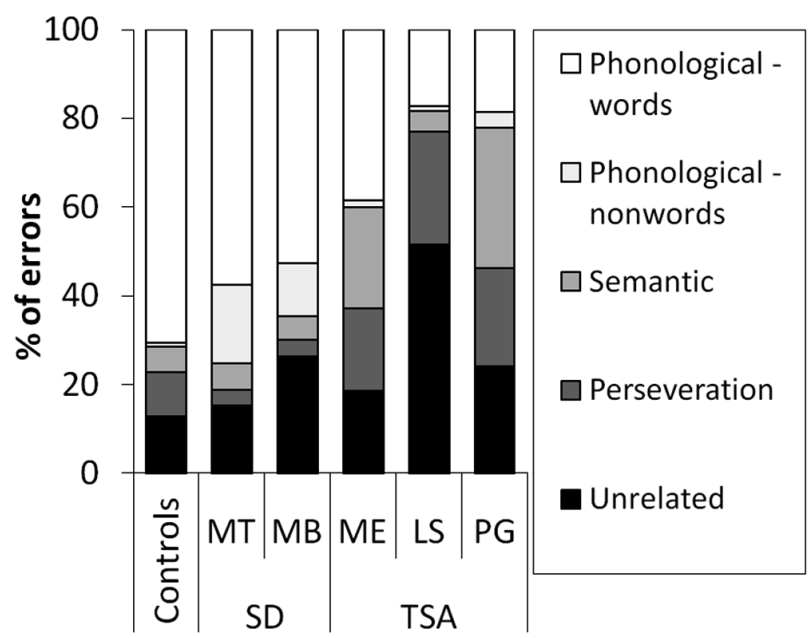

Figure 4. Classification of intrusion errors.

shows that, despite the improvement in item recall, the patients continued to make order errors on $\mathrm{G}$ - lists. For ME the order error rate remained as high as for the original lists, despite her improvement in item recall. Errors were less frequent in the other two patients, but remained at higher levels than those observed in the SD cases. In addition, all three patients made more errors to $\mathrm{G}-$ lists than to $\mathrm{G}+$ lists, $\chi^{2}(d f=1)>$ $5.7, p<.05$. Finally, Figure 7 shows the proportion of responses that were grammatically formed. ME continued to give grammatically well-formed responses to almost all lists. PG and LS showed some sensitivity to list structure with the new materials but persisted in giving grammatical responses to around half of the $\mathrm{G}$ - lists, indicating that activation of familiar syntactic structures continued to dominate their responses. 


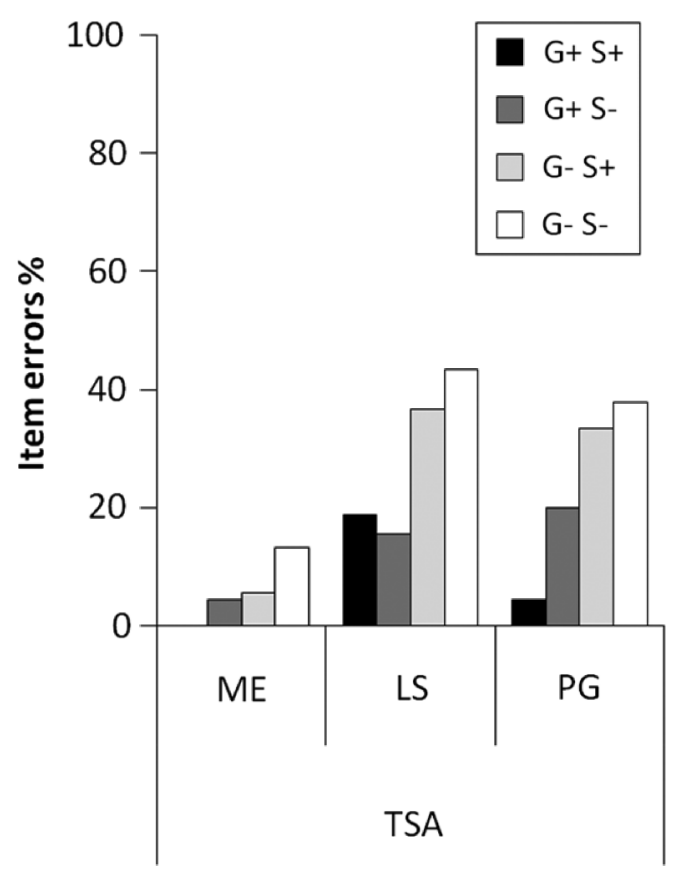

Figure 5. Item errors for shorter lists.

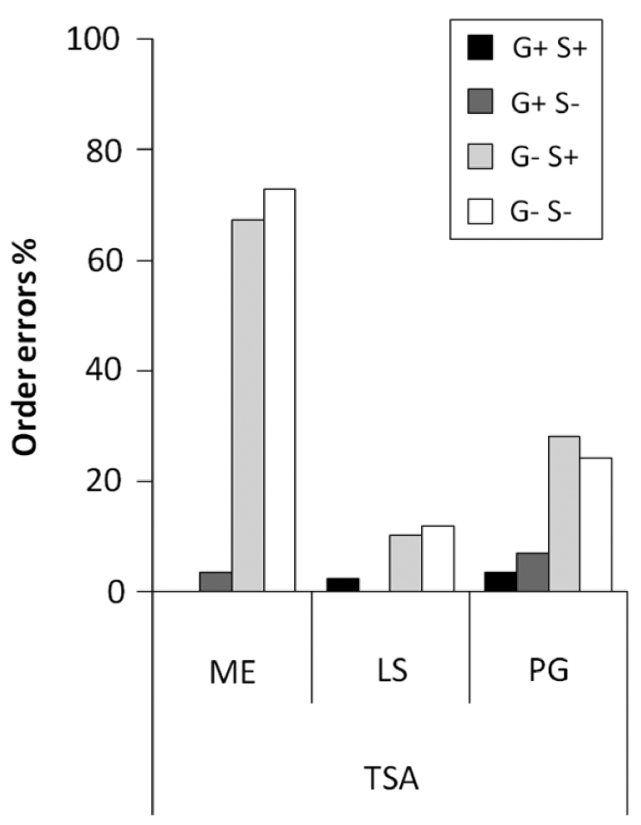

Figure 6. Order errors for shorter lists. 


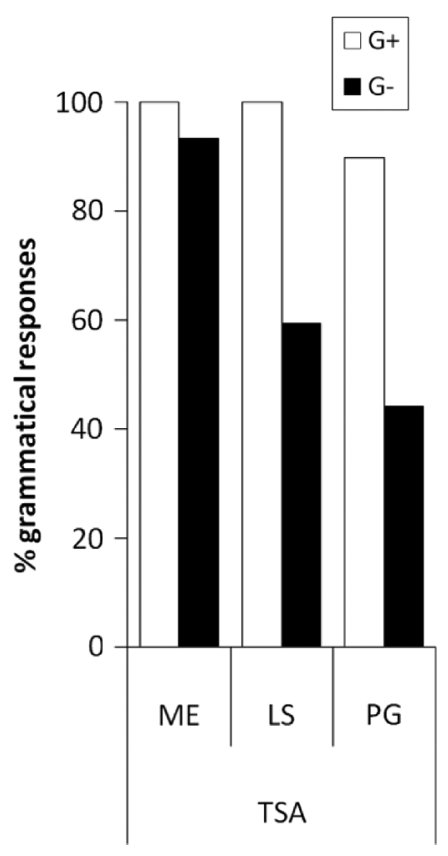

Figure 7. Grammatical structure of responses for shorter lists.

\section{DISCUSSION}

Most contemporary theories of verbal STM agree that activation of language representations makes an important contribution to verbal short-term recall. Some models, including Baddeley's original conception of the episodic buffer, take the view that executive control is a necessary prerequisite for this linguistic contribution (Baddeley, 2000; Majerus, 2009; Ruchkin et al., 2003). However, recent studies in healthy participants suggest that naturalistic sentences enjoy a significant recall advantage over random lists even when executive resources are occupied with a demanding secondary task (Baddeley et al., 2009; Jefferies, Lambon Ralph, et al., 2004). These findings support an alternative view in which the activation of stored linguistic knowledge is a basic and automatic element of STM, and that executive demands arise when the material to be remembered conflicts with this activation. We tested this hypothesis by investigating STM in three TSA patients with impaired executive regulation of linguistic knowledge. These individuals showed robust "sentence superiority" effects despite their executive deficits: they were better at recalling words that shared a common semantic theme and were much more likely to produce words in the correct sequence when their order conformed to a familiar syntactic structure. Moreover, these effects were larger than those observed in healthy participants. Most strikingly, the patients had great difficulty producing sequences of words that were not grammatically correct, even when the task required this. When presented with novel, scrambled word lists, they often distorted their order to better fit with their previous linguistic experience, suggesting that activated long-term language knowledge dominated their recall.

These findings suggest that a key role of executive control in verbal STM tasks may be to prevent activation of stored linguistic knowledge from unduly influencing responses when it is inappropriate. This role has rarely been identified previously 
in STM studies. In healthy participants executive control is typically associated with working memory tasks in which items are manipulated or processed prior to responding, but is often thought to play a less-prominent role in simple reproduction tasks like the one used here (Engle, Tuholski, Laughlin, \& Conway, 1999; Smith \& Jonides, 1999). This may be because most studies of verbal STM have deliberately employed random word lists with the intention of minimising any contribution of linguistic LTM. Under such circumstances participants may adopt a strategy of encoding only the phonological characteristics of the list and rely less on activated long-term knowledge (Campoy \& Baddeley, 2008). Future studies that directly compare random word lists with sentences under executively demanding conditions may be useful in better understanding this phenomenon.

We also investigated the effects of semantic and syntactic structure on STM in two SD patients. They exhibited a different pattern of performance from the TSA cases, even when overall levels of recall were taken into account. They had no deficit for materials that violated semantic and syntactic constraints but instead showed a selective impairment for meaningful, well-formed sentences. This erosion of the sentence superiority effect is consistent with the gradual breakdown of semantic knowledge in this patient group and, in particular, with the "semantic binding" account of lexical effects in STM (Jefferies et al., 2006; Patterson et al., 1994). On this view there are two sources of constraint on phonological processing that can support verbal STM. First, the phonological elements of a word are strongly associated with one another because they are co-activated whenever the word is encountered. The phonological system itself therefore develops pattern-completion properties that aid the maintenance of the phonological forms of familiar words. Second, whenever the phonological representation of a word is activated, its semantic representation is also activated. The association formed between a word's semantic representation and its phonological representation then serves as an additional source of constraint, helping to ensure that the correct configuration of phonological elements remains active. This theory is supported by distributed, connectionist models of language in which the primary systems of semantics and phonology interact to give rise to linguistic performance across a range of tasks (Patterson \& Lambon Ralph, 1999; Plaut \& Kello, 1999). In these models "lexical" effects, such as the superior recall for meaningful words, emerge from the interactions between the semantic and phonological representations (Dilkina, McClelland, \& Plaut, 2010).

As in previous studies (Hoffman et al., 2009; Jefferies et al., 2006; Jefferies, Jones, et al., 2004; Patterson et al., 1994), the semantic deficit in the SD cases led to frequent phonological errors, supporting the theory that semantic knowledge plays an important role in preserving the phonological forms of words held in STM. Similar phonological errors occur when healthy participants repeat word lists that contain meaningless nonwords (Hoffman et al., 2009; Jefferies et al., 2006; Treiman \& Danis, 1988). Phonological errors were much less prominent in the TSA patients, suggesting that this form of linguistic support can persist when executive function is disrupted. In contrast, memory for word order was severely impaired in TSA patients but entirely preserved in the SD patients, confirming that the TSA patients' inability to deal with the novel word order of the scrambled word lists was not a general effect of comprehension impairment.

As a final point, we note that while both item and order memory were impaired in our TSA patients, the largest effects of linguistic constraints were seen in order memory. One possible explanation is that the patients had damage to a dedicated system 
that ensures words are selected for output in the correct sequence (N. Burgess \& Hitch, 1999; Majerus, 2009; Martin \& Gupta, 2004). However, a deficit for order memory per se is unlikely, as digit span, a task that depends heavily on order memory, was intact in all patients. It is more likely that the striking effects of syntactic structure reflect the strong constraints syntax places over the sequencing of words in normal speech. Although STM studies typically eschew syntactically structured materials in the interests of experimental parsimony, there is little doubt that syntax is central to speech comprehension. Indeed, a number of brain regions respond more strongly to syntactically meaningful word sequences, including areas of inferior frontal, anterior temporal and inferior parietal cortex (e.g., Friederici, Ruschemeyer, Hahne, \& Fieback, 2003; Humphries, Binder, Medler, \& Liebenthal, 2006). When processing speech the language system therefore has strong expectation of a syntactically appropriate sequence, and when this expectation is violated there is conflict between the patient's internal representations of expected word order and the sequence that was actually presented. The challenge under these circumstances is to inhibit the strong activation of the expected word order and instead direct attention to maintaining the actual sequence. Patients with impaired executive control and regulation of internal representations were unable to do this, with the result that their responses were driven not by the unusual sequences they heard during the task, but rather by strong internal representations of legitimate word sequences acquired through a lifetime of linguistic experience. We propose that this unusual behaviour is best conceptualised not as a specific deficit in STM for serial order or syntactic processing, but as a more general impairment in executive regulation of knowledge. This regulation impairment has the potential to explain both the impaired semantic processing in these individuals (Corbett et al., 2009; Hoffman et al., 2010; Noonan et al., 2010) and their verbal STM deficits (Hoffman, Jefferies, \& Lambon Ralph, 2011; Jefferies, Hoffman, et al., 2008).

\section{REFERENCES}

Acheson, D. J., \& MacDonald, M. C. (2009). Verbal working memory and language production: Common approaches to the serial ordering of information. Psychological Bulletin, 135, 50-68.

Albert, M., Goodglass, H., Helms, N. A., Rubens, A. B., \& Alexander, M. N. (1981). Clinical aspects of dysphasia. New York/Berlin: NSpringer-Verlag.

Baddeley, A. D. (2000). The episodic buffer: A new component of working memory? Trends in Cognitive Sciences, 4(11), 417-423.

Baddeley, A. D., \& Hitch, G. J. (1974). Working memory. In G. Bower (Ed.), The psychology of learning and motivation (Vol. 8, pp. 47-90). New York: Academic Press.

Baddeley, A. D., Hitch, G. J., \& Allen, R. J. (2009). Working memory and binding in sentence recall. Journal of Memory and Language, 61(3), 438-456.

Badre, D., \& Wagner, A. D. (2002). Semantic retrieval, mnemonic control, and prefrontal cortex. Behavioral and Cognitive Neuroscience Reviews, 1, 206-218.

Bourassa, D. C., \& Besner, D. (1994). Beyond the articulatory loop: A semantic contribution to serial order recall of subspan lists. Psychonomic Bulletin \& Review, 1(1), 122-125.

Bozeat, S., Lambon Ralph, M. A., Patterson, K., Garrard, P., \& Hodges, J. R. (2000). Non-verbal semantic impairment in semantic dementia. Neuropsychologia, 38(9), 1207-1215.

Brener, R. (1940). An experimental investigation of memory span. Journal of Experimental Psychology, 26, $467-482$.

Burgess, N., \& Hitch, G. J. (1999). Memory for serial order: A network model of the phonological loop and its timing. Psychological Review, 106(3), 551-581.

Burgess, P., \& Shallice, T. (1997). The Hayling and Brixton tests. Suffolk, UK: Thames Valley Test Company.

Campoy, G., \& Baddeley, A. (2008). Phonological and semantic strategies in immediate serial recall. Memory, 16(4), 329-340. 
Corbett, F., Jefferies, E., Ehsan, S., \& Lambon Ralph, M. A. (2009). Different impairments of semantic cognition in semantic dementia and semantic aphasia: Evidence from the non-verbal domain. Brain, $132,2593-2608$.

Cowan, N. (1995). Attention and memory: An integrated framework. New York: Oxford University Press.

Crawford, J. R., \& Howell, D. C. (1998). Comparing an individual's test score against norms derived from small samples. The Clinical Neuropsychologist, 12, 482-486.

Dilkina, K., McClelland, J. L., \& Plaut, D. C. (2010). Are there mental lexicons? The role of semantics in lexical decision. Brain Research, 1365, 66-81.

Engle, R. W., Tuholski, S. W., Laughlin, J. E., \& Conway, A. R. A. (1999). Working memory, short-term memory, and general fluid intelligence: A latent-variable approach. Journal of Experimental Psychology: General, 128(3), 309-331.

Friederici, A. D., Ruschemeyer, S. A., Hahne, A., \& Fieback, C. J. (2003). The role of left inferior frontal and superior temporal cortex in sentence comprehension: Localizing syntactic and semantic processes. Cerebral Cortex, 13, 170-177.

Garrard, P., \& Carroll, E. (2006). Lost in semantic space: A multi-modal, non-verbal assessment of feature knowledge in semantic dementia. Brain, 129, 1152-1163.

Gathercole, S. E., Willis, C., Emslie, H., \& Baddeley, A. D. (1991). The influences of number of syllables and wordlikeness on children's repetition of nonwords. Applied Psycholinguistics, 12(3), 349-367.

Goodglass, H. (1983). The assessment of aphasia and related disorders (2nd edition). Philadelphia: Lea \& Febiger.

Hamilton, A. C., \& Martin, R. C. (2005). Dissociations among tasks involving inhibition: A single-case study. Cognitive Affective \& Behavioral Neuroscience, 5(1), 1-13.

Hodges, J. R., \& Patterson, K. (2007). Semantic dementia: A unique clinicopathological syndrome. Lancet Neurology, 6(11), 1004-1014.

Hodges, J. R., Patterson, K., Oxbury, S., \& Funnell, E. (1992). Semantic dementia: Progressive fluent aphasia with temporal lobe atrophy. Brain, 115, 1783-1806.

Hoffman, P., Jefferies, E., Ehsan, S., Jones, R. W., \& Lambon Ralph, M. A. (2009). Semantic memory is key to binding phonology: Converging evidence from immediate serial recall in semantic dementia and healthy participants. Neuropsychologia, 47(3), 747-760.

Hoffman, P., Jefferies, E., \& Lambon Ralph, M. A. (2010). Ventrolateral prefrontal cortex plays an executive regulation role in comprehension of abstract words: Convergent neuropsychological and rTMS evidence. Journal of Neuroscience, 46, 15450-15456.

Hoffman, P., Jefferies, E., \& Lambon Ralph, M. A. (2011). Remembering 'zeal' but not 'thing': Reverse frequency effects as a consequence of deregulated semantic processing. Neuropsychologia, 49, 580-584.

Hoffman, P., Rogers, T. T., \& Lambon Ralph, M. A. (in press). Semantic diversity accounts for the "missing" word frequency effect in stroke aphasia: Insights using a novel method to quantify contextual variability in meaning. Journal of Cognitive Neuroscience.

Hulme, C., Roodenrys, S., Schweickert, R., Brown, G. D. A., Martin, S., \& Stuart, G. (1997). Wordfrequency effects on short-term memory tasks: Evidence for a redintegration process in immediate serial recall. Journal of Experimental Psychology: Learning, Memory and Cognition, 23(5), 1217-1232.

Humphries, C., Binder, J. R., Medler, D. A., \& Liebenthal, E. (2006). Syntactic and semantic modulation of neural activity during auditory sentence comprehension. Journal of Cognitive Neuroscience, 18, 665-679.

Jefferies, E., Bott, S., Ehsan, S., \& Lambon Ralph, M. A. (2011). Phonological learning in semantic dementia. Neuropsychologia, 49(5), 1208-1218.

Jefferies, E., Frankish, C., \& Lambon Ralph, M. A. (2006). Lexical and semantic binding in verbal shortterm memory. Journal of Memory and Language, 54, 81-98.

Jefferies, E., Hoffman, P., Jones, R., \& Lambon Ralph, M. A. (2008). The impact of semantic impairment on verbal short-term memory in stroke aphasia and semantic dementia: A comparative study. Journal of Memory and Language, 58(1), 66-87.

Jefferies, E., Jones, R., Bateman, D., \& Lambon Ralph, M. A. (2004). When does word meaning affect immediate serial recall in semantic dementia? Cognitive, Affective and Behavioral Neuroscience, 4(1), $20-42$.

Jefferies, E., Jones, R. W., Bateman, D., \& Lambon Ralph, M. A. (2005). A semantic contribution to nonword recall? Evidence for intact phonological processes in semantic dementia. Cognitive Neuropsychology, 22(2), 183-212.

Jefferies, E., \& Lambon Ralph, M. A. (2006). Semantic impairment in stroke aphasia vs. semantic dementia: A case-series comparison. Brain, 129, 2132-2147. 
Jefferies, E., Lambon Ralph, M. A., \& Baddeley, A. D. (2004). Automatic and controlled processing in sentence recall: The role of long-term and working memory. Journal of Memory and Language, 51(4), 623-643.

Jefferies, E., Patterson, K., Jones, R. W., Bateman, D., \& Lambon Ralph, M. A. (2004). A categoryspecific advantage for numbers in verbal short-term memory: Evidence from semantic dementia. Neuropsychologia, 42(5), 639-660.

Jefferies, E., Patterson, K., \& Lambon Ralph, M. A. (2008). Deficits of knowledge versus executive control in semantic cognition: Insights from cued naming. Neuropsychologia, 46, 649-658.

Majerus, S. (2009). Verbal short-term memory and temporary activation of language representations: The importance of distinguishing item and order information. In A. Thorn \& M. Page (Eds.), Interactions between short-term and long-term memory in the verbal domain (pp. 244-276). Hove, UK: Psychology Press.

Majerus, S., Norris, D., \& Patterson, K. (2007). What does a patient with semantic dementia remember in verbal short-term memory? Order and sound but not words. Cognitive Neuropsychology, 24, 131-151.

Martin, N., \& Gupta, P. (2004). Exploring the relationship between word processing and verbal short-term memory: Evidence from associations and dissociations. Cognitive Neuropsychology, 21, 213-228.

Martin, N., \& Saffran, E. M. (1997). Language and auditory-verbal short-term memory impairments: Evidence for common underlying processes. Cognitive Neuropsychology, 14(5), 641-682.

Meteyard, L., \& Patterson, K. (2009). The relation between content and structure in language production: An analysis of speech errors in semantic dementia. Brain and Language, 110(3), 121-134.

Miller, G. A., \& Selfridge, J. A. (1950). Verbal context and the recall of meaningful material. American Journal of Psychology, 63, 176-185.

Noonan, K. A., Jefferies, E., Corbett, F., \& Lambon Ralph, M. A. (2010). Elucidating the nature of deregulated semantic cognition in semantic aphasia: Evidence for the roles of the prefrontal and temporoparietal cortices. Journal of Cognitive Neuroscience, 22, 1597-1613.

Patterson, K., Graham, N., \& Hodges, J. R. (1994). The impact of semantic memory loss on phonological representations. Journal of Cognitive Neuroscience, 6(1), 57-69.

Patterson, K., \& Lambon Ralph, M. A. (1999). Selective disorders of reading? Current Opinion in Neurobiology, 9(2), 235-239.

Patterson, K., \& MacDonald, M. C. (2006). Sweet nothings: Narrative speech in semantic dementia. In S. Andrews (Ed.), From inkmarks to ideas: Current issues in lexical processing (pp. 299-317). Hove, UK: Psychology Press.

Patterson, K., Nestor, P. J., \& Rogers, T. T. (2007). Where do you know what you know? The representation of semantic knowledge in the human brain. Nature Reviews Neuroscience, 8(12), 976-987.

Plaut, D. C., \& Kello, C. T. (1999). The emergence of phonology from the interplay of speech comprehension and production: A distributed connectionist approach. In B. MacWhinney (Ed.), The emergence of language. Mahwah, NJ: Lawrence Erlbaum Associates Inc.

Rodd, J. M., Davis, M. H., \& Johnsrude, I. S. (2005). The neural mechanisms of speech comprehension: fMRI studies of semantic ambiguity. Cerebral Cortex, 15(8), 1261-1269.

Rogers, T. T., Lambon Ralph, M. A., Garrard, P., Bozeat, S., McClelland, J. L., Hodges, J. R., et al. (2004). Structure and deterioration of semantic memory: A neuropsychological and computational investigation. Psychological Review, 111(1), 205-235.

Romani, C., McAlpine, S., \& Martin, R. C. (2008). Concreteness effects in different tasks: Implications for models of short-term memory. Quarterly Journal of Experimental Psychology, 61, 292-323.

Ruchkin, D. S., Grafman, J., Cameron, K., \& Berndt, R. S. (2003). Working memory retention systems: A state of activated long-term memory. Behavioral and Brain Sciences, 26(6), 709-777.

Saint-Aubin, J., \& Poirier, M. (1999). Semantic similarity and immediate serial recall: Is there a detrimental effect on order information? Quarterly Journal of Experimental Psychology, 52 A(2), 367-394.

Schweickert, R. (1993). A multinomial processing tree model for degradation and redintegration in immediate recall. Memory \& Cognition, 21(2), 168-175.

Smith, E. E., \& Jonides, J. (1999). Storage and executive processes in the frontal lobes. Science, 283(5408), 1657-1661.

Snowden, J. S., Goulding, P. J., \& Neary, D. (1989). Semantic dementia: A form of circumscribed cerebral atrophy. Behavioural Neurology, 2, 167-182.

Soni, M., Lambon Ralph, M. A., Noonan, K., Ehsan, S., Hodgson, C., \& Woollams, A. M. (2009). "L" is for tiger: Effects of phonological (mis)cueing on picture naming in semantic aphasia. Journal of Neurolinguistics, 22(6), 538-547. 
Thompson-Schill, S. L., D’Esposito, M., Aguirre, G. K., \& Farah, M. J. (1997). Role of left inferior prefrontal cortex in retrieval of semantic knowledge: A re-evaluation. Proceedings of the National Academy of Sciences of the United States of America, 94, 14792-14797.

Treiman, R., \& Danis, C. (1988). Short-term memory errors for spoken syllables are affected by the linguistic structure of the syllables. Journal of Experimental Psychology: Learning, Memory and Cognition, 14(1), 145-152.

Wechsler, D. (1987). Wechsler memory scale: Revised (WMS-R). New York: Psychological Corporation. 